\title{
DAYLIGHTING IN ATRIUM BUILDING: A STUDY OF THE INFLUENCE OF ATRIUM FAÇADE DESIGN
}

\author{
S. SAMANT \\ Department of Architecture and Built Environment, University of Nottingham, UK.
}

\begin{abstract}
Atrium spaces have the potential to make vital contribution to the sustainable strategy and consequently ecodynamics of a building. The environmental benefits in terms of daylight, natural ventilation, and heating that an atrium offers are widely recognised. Daylight availability in an atrium space is generally high; however, this may not necessarily be true for the adjoining spaces. Previous studies indicate that the daylight performance of the adjoining spaces can be improved through the design of atrium facades, whereby there is a progressive increase in the fenestration from the upper to the lower floors. Therefore, this paper seeks to systematically investigate the effects of different atrium façades design characterised by varied distribution of fenestrations on daylight (DFs) in an atrium and horizontal penetration of daylight in its adjoining spaces under overcast sky conditions for a four sided, top-lit, square atrium building of Well Index (WI) 1.25. Studies were undertaken using computer simulation software programs ECOTECT and RADIANCE with the objective of understanding the influence of facades and providing guidelines for facade design to create optimal daylighting conditions in the adjoining spaces. Three main curves were developed, each of which included five options of $20 \%, 30 \%$, $40 \%, 50 \%$ and $60 \%$ openings on top floor with a progressive increase in openings and $100 \%$ opening on the ground floor. Results demonstrate that façade compositions have a very limited influence on daylight in the adjoining spaces offering very little benefits to the lower floors, where daylight is critical. But increase in opening on the top floor may increase DFs significantly on the top two floors. For this study, the option of $60 \%$ opening on top floor with progressive increase to $100 \%$ opening on ground floor provided the best results. Keywords: adjoining spaces, atrium, daylighting strategies, facade design, fenestration distribution
\end{abstract}

\section{INTRODUCTION}

Atrium spaces can be employed to create sustainable solutions in a variety of building types and therefore make significant contributions to the eco-dynamics of a building. If appropriately designed, atria present huge environmental benefits in terms of daylight, natural ventilation, and heating. The importance of daylight in an atrium's environmental performance has led to several investigations of daylighting in atria and their adjoining spaces. Although the daylight potential of an atrium has been recognised widely, atrium buildings have been unable to successfully utilise daylight in spaces adjoining the atria. Daylight levels within the atrium space are generally sufficiently high. However, this may not be the case for spaces adjoining the atria, where daylight varies significantly with every floor level. Rooms on the top floors can be over-lit and suffer from glare while daylight levels on the lower floors can be low, particularly in tall/deep atria.

Daylight performance (quantity and distribution) of an atrium and its adjoining spaces is complex and affected by five elements:

The predominant sky conditions and external daylight availability.

- The roof configuration which affects the quantity and direction of light. The fenestration system will control the intensity and spatial distribution of light entering the atrium. The net transmittance of the fenestration will vary with the roof structure and geometry; glazing system - its orientation and type; shading systems.

- The basic atrium type - its geometry and relative proportions; the size of the atrium and its configuration. 
- The atrium enclosing surfaces which determine how much light is going to be transmitted to the adjacent spaces, or reflected down towards the lower floors. This includes atrium facade design, its surface reflectances, window size/positioning, use of innovative daylighting systems (light shelves, light scoops); and atrium floor reflectances.

- Design properties of the adjacent spaces, including their geometry, surface reflectances, room furnishings and furniture layout.

\section{BACKGROUND}

In an atrium well, daylight factor (DF) comprises of the sky component (SC) and the internally reflected component (IRC) from the atrium's enclosing surfaces (walls and floor). Therefore, wall reflectance has a direct and significant impact on inter-reflectance occurring inside the light well and determines the distribution of light in the space, and the amount of light which reaches the lower levels. CIBSE Code for Interior Lighting (CIBSE [1]) recommended that reflectances of the atrium well facades should also be as high as possible to improve daylight in the adjoining space. However, the amount of increase would depend on the atrium form.

Letherman and Wright [2] rightly point out that in high Well Index (WI) atria, the relative surface area of the atrium's walls is high thus the potential for a large IRC is also high. However, as the view factor between the atrium's walls and sky vault is small, illuminance and consequently wall luminance are low. As the WI decreases, the IRC increases due to the increasing relative size of the atrium floor with respect to the atrium walls of higher luminance due to the increase in view factor with the sky vault. As the WI becomes very low however, the IRC decreases with the wall area becoming too small for an IRC of any significant magnitude.

For atrium surfaces comprising of different materials (glazed and opaque), an area-weighted reflectance is used to calculate IRC, where each material reflectance is multiplied with the area of its use and these figures are summed up and divided by the total area. Although this value gives an impression of the daylight availability, it does not depict a picture of how this daylight is distributed in the space due to the arrangement of windows and various materials within these surfaces. 'Despite the simplicity of their models, Aizlewood et al. [3] failed to correlate measured IRC values with calculated values, demonstrating the complex and as yet poorly understood behaviour of reflected flux, particularly when highly reflective surfaces are used' (Sharples and Lash [4]).

\section{LITERATURE REVIEW}

Oretskin [5] showed that for an indexed depth of 1.0, increasing the wall reflectances from 0.2 to 0.5 will double the vertical illuminance. Navvab and Selkowitz [6] looked at the effects of a change in atrium wall reflectivity from 0.50 to 0.86 for five fenestration systems under several sun and sky conditions in a five storey atrium. The study showed that atrium wall glazing characteristics influence the flux distribution and intensity as a function of its position in the atrium. Measurements indicated that daylight on a vertical atrium wall is normally less than $20 \%$ of the exterior horizontal value once one moves below two or three floors depth indicating that task illuminance within an adjacent space would also be low. Cartwright [7] also showed the effect of varying wall reflectances on vertical illuminances as a function of well-indexed depth. Aschehoug [8] produced IRC information for walls of $40 \%$ and $90 \%$ diffuse reflectance, within a well index range from 0.75 to 2.0 while Liu et al. [9] gave computer-predicted effect of varying wall reflectances $(30 \%, 45 \%, 60 \%)$ on DF at the base of the atrium, as a function of WI in a four sided atrium.

Several authors (Aschehoug [8]; Cole [10]; Boubekri [11]) suggest that the proportion of window area should vary between the floors of the atrium. Since most daylight is available at the top of the 
atrium, adjoining spaces need the smallest windows to achieve desired daylight levels. A progressive increase in the amount of openings from upper to the lower floors can lead to higher DFs available at the bottom of the atrium.

Aschehoug [8] studied daylight distribution in adjoining spaces with windows facing a glazed street of infinite length. Main parameters that govern daylight conditions were systematically altered, which included street width/building height ratios, window sizes, and façade reflectances. This study presented an 'optimum' glazing percentage for the facades facing a glazed space with $50 \%$ glazing on the 4 th floor, $60 \%$ glazing on the 3rd floor, $70 \%$ glazing on the 2 nd floor and $100 \%$ glazing on the 1 st floor to give quite similar daylight conditions in rooms on all floors in the adjacent buildings.

Willbold-Lohr [12] studied different facade apertures in square shaped atria with a well index ranging from 0.5 to 2.0 . Completely white façade (reflectance 0.7 ), facade with $50 \%$ window/wall ratio (average reflectance 0.4 ), only glazing (average reflectance 0.1 ), completely black facade (reflectance 0.05) had been tested with glazing material. The study concluded that a facade aperture with $50 \%$ window openings will reduce the contribution of the IRC by half and having only glazed walls as separation between the office and the atrium the IRC is reduced to $1 / 3$ of the white walls, and almost reduced to the contribution of the skylight alone.

Cole [10] undertook scale model study to examine daylight factor distribution in the adjacent spaces of the ground floor, third and fifth floor, respectively, of an open, square, five storeys, atrium building with $100 \%, 50 \%$, and variable openings into adjacent spaces. The study demonstrates that the variable opening option of $100 \%$ on Ground, $80 \%$ on 2 nd, $60 \%$ on $3 \mathrm{rd}, 40 \%$ on 4 th and $20 \%$ on 5 th floor is the most effective in terms of bringing daylight on the lower floors of adjoining spaces in atrium buildings, where it is most needed.

Iyer [13] studied effect of five wall reflectances $(90 \%, 85 \%, 75 \%, 50 \%, 25 \%)$ in a rectangular top-lit atrium $(\mathrm{WI}=1.95)$ without any roof glazing for $25 \%, 50 \%$ and $75 \%$ openings in the wall. It was concluded that the difference in DF between any two points in the adjacent space is greater when the surface reflectivity of the wall is higher. There is more uniform distribution of DF in adjoining spaces for $25 \%$ atrium wall openings than $50 \%$ and $75 \%$ openings due to the increased inter reflectance of light down the atrium well and into the side spaces. However, large openings give greater illumination and a wide range of illumination values.

Boubekri [11] illustrated the effect of wall reflectance 56\%, 42\%, $28 \%$ and $14 \%$ that corresponded to $0 \%, 25 \%, 50 \%$ and $75 \%$ glazing, respectively, on daylight distribution under roof cover with horizontal glazing. As the wall reflectance increased from $14 \%$ to $56 \%$, the overall DF on the walls at the upper level increased from $23 \%$ to $37 \%$ and from $11 \%$ to $23 \%$ at the lower level. Aizlewood et al. [3] carried out parametric studies of the atrium surface reflectances (74\%, $47 \%, 33 \%, 6 \%$ ), the atrium geometry, and the geometry of the adjoining spaces and concluded that as the WI increases, DF at the base of the atrium falls rapidly for surfaces of low or middling reflectances.

Undertaking physical model studies for a linear atrium, Matusiak et al. [14] evidenced that varying glazing area or glazing type results in a small but significant increase in daylight on the atrium floor, and improves balance of lighting in the adjoining spaces. However, changing glazing type was considered to be a less flexible option due to limited availability of glazing that might have similar colour but different transmittance properties. They present DF measurements on atrium surfaces and on vertical and horizontal planes in the adjoining spaces. They gave formulas for the luminance distribution on linear atrium facades, and simple rules of thumb for estimating daylight factors in the adjoining spaces. 
Horizontal daylight factors in the adjacent rooms will depend on the vertical daylight factor on the atrium facades (on the middle height of the window) and on the relation Agl/Afl where Agl is the glass area and Afl is the floor area of the room. The following rules of thumb were tried:

DFmin $=0.25 \times$ DFvert $\times(\mathrm{Agl} / \mathrm{Afl}) \times(\tau / \tau$ clear $)$ rule 1

DFmean $=0.5 \times$ DFvert $\times(\mathrm{Agl} / \mathrm{Afl}) \times(\tau / \tau$ clear $)$ rule 2

The correction factor $\tau / \mathcal{Z}$ clear is used, where $\tau$ is the transmission factor of the actual glazing and $\tau$ clear is the transmission factor of the double clear glass. The comparisons of measured and calculated daylight factors show that the proposed rules of thumb give results with an accuracy of $30 \%$.

Sharples and Mahambrey [15] examined the effect of different distribution patterns of atrium wall reflectances (representing atrium wall surfaces in real buildings) on DF at various positions in the well of a square, four-sided, top-lit atrium under Commission Internationale de l'Eclairage (CIE) overcast sky that is used for daylighting calculations in temperate climates. The study concluded that atrium surfaces with wide bands of different reflectance values affect DFs at the base of the atrium. However, if these bands are narrow, DFs are not significantly altered by different reflectance distributions. The introduction of specular glass surfaces into the atrium produces a consistent increase in the DF and Atrium Reflected Component (ARC) values, but does not alter the general conclusions drawn from the measurements with just the diffuse surfaces.

As an extension of this study, Samant and Sharples [16] compared average daylight factor (ADF) values on the floor of the atrium well with those obtained from Littlefair's [17] average daylight factor at the base of an atrium, ADFb formula:

$$
\mathrm{ADFb}=\frac{\mathrm{WTg} \operatorname{Tr} \theta}{\mathrm{S}(1-\mathrm{R} 2)}
$$

where, $\mathrm{W}$ is the area of the of the atrium roof opening $\left(\mathrm{m}^{2}\right)$; $\mathrm{Tg}$ is the diffuse visible transmittance of the glazing (corrected for dirt); $\mathrm{Tr}$ is an atrium roof structure blockage factor; $\mathrm{S}$ is the total area of all the atrium surfaces (roof, windows, walls and floor) in $\mathrm{m}^{2} ; \mathrm{R}$ is the average, area-weighted reflectance of all the surfaces used to estimate $S$; and $\theta$ is the angle of visible sky in degrees as measured in Fig. 1.

Results from the study showed that the ADF is affected by the reflectance distributions of the atrium surfaces. The expression for atrium floor ADF (Littlefair [17]) gave good agreement with measured data from this study for all-black surfaces but underestimated values, by nearly $20 \%$ for white walled atrium. The expression underestimated IRC by $8-15 \%$ for an atrium floor with a number of bands of different reflectances. Therefore, for mixed reflectance and predominantly light coloured atria, ADF predicted by the equation (Littlefair [17]) could be multiplied by a factor of 1.1 and 1.2 , respectively.

As an extension of Sharples and Mahambrey [15], Sharples and Lash [18] examined the effects of atrium wall distribution patterns on vertical DFs at various heights for central positions. The different

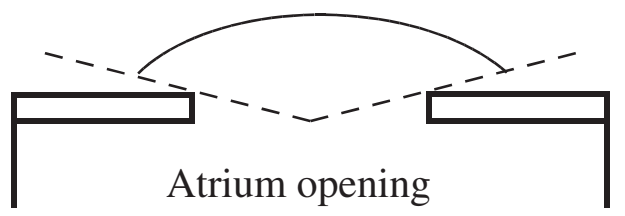

Figure 1: Definition of visible sky angle $\theta$. 
distributions of reflectances were found to have very little effect on vertical DFs and IRC low down in the atrium well. For some of the higher measurement locations large differences were observed between the different reflectance distributions. As the number of bands increased and the bands became narrower, DFs achieved were similar to those predicted by the standard formula using areaweighted reflectance of the atrium.

Matusiak [19] undertook full-scale studies of a small tea-room in Norway with the objective of assessing the impact of artificial lighting/daylighting and reflectances on the size impression of the room. The study concluded that the size impression of a room changes with higher reflection factors and/or illuminances on the surfaces in the room make them appear more distant, making the room appear larger. However, this effect is only achieved with small luminance contrasts between surfaces making improved luminance distribution very important.

Calcagni and Paroncini [20] provided a relationship between the main architectural components of an atrium (geometry, material properties, fenestration system, atrium roof) and daylight conditions in the adjoining space and on the atrium floor. Eleven atrium (square and rectangular) cases, characterized by a different WI $(0.2-1.47)$, and atrium wall reflectance $(10 \%, 30 \%, 50 \%, 70 \%, 90 \%)$ were investigated under the CIE overcast sky. Simplified formulas (for atrium with and without roof) derived from Radiance were developed for preliminary prediction of horizontal DF on the atrium floor and in the adjacent rooms at a distance of $4 \mathrm{~m}$. The study evidenced that the atrium roof cuts DF by about $45 \%$ in the area adjacent to the atrium, and reduces the influence of surface reflectances as well as WI giving quite similar DF values of between 1 and 2\%. As the WI increases from 0.2 to $0.75 \mathrm{DF}$ values drop sharply, however when WI increases from 0.75 to 1.29 , quite similar DF values are achieved. This suggests that WI $>1.29$ would have limited influence on DF in spaces adjoining the atria; this is in agreement with the findings of Samant and Yang's [21] study. Additionally, whilst keeping the height same, increasing the length of the atrium increases the light-admitting area (or reduces WI) and consequently DF. The study shows that although increase in wall reflectance from $30 \%$ to $70 \%$ increases DF by about $4.8 \%$ in the workspaces for several WI, it does not produce a significant improvement in the DF on the ground floor due to large windows with high transmittance limiting surfaces that could reflect light.

Samant and Yang [21] made parametric changes to the distribution of reflectances of diffuse atrium well surfaces in atriums with a WI 0.5, 1.0 and 1.5. In agreement with Calcagni and Paroncini [20], it was concluded that the well reflectance distributions have limited influence on daylight distribution in shallow or wide medium sized atria but have practically no influence in tall atria.

Lau and Duan [22] examined the effect of different types and arrangement of specular surfaces in atria on daylighting in adjacent spaces. The study demonstrated that adding specular surfaces $(23 \%$, $47 \%$ and $90 \%$ reflectance) to atrium parapet walls increased illuminance levels in these spaces, and that top floor parapet walls alone resulted in higher DF $(\sim 25 \%)$ at the atrium floor and that there was no need to add specular surfaces to parapets at every floor level.

\section{METHODOLOGY}

Several studies indicate the potential to improve daylight in adjoining spaces through glazing distributions of progressive increase in the amount of openings from the upper to the lower floors in medium scale atria. Aschehoug [8] recommended optimum glazing ratios for a glazed street of infinite length, whilst Calcagni and Paroncini [20] provided relationship between surface reflectances in an atrium and daylight conditions in the adjoining space and on the atrium floor. In agreement with Samant and Yang [21], Calcagni and Paroncini [20] also confirmed that glazing/reflectance distributions have influence in medium scale buildings between WI of 0.75-1.29. Therefore, the aim of the experiments was to systematically study parametrically the effects of different glazing distributions 
on daylight (DF) in the atrium and horizontal penetration of daylight in its adjoining spaces under overcast sky conditions. The objective was to provide optimum glazing ratios for a four sided, toplit, square shaped, medium size atrium building.

The experiments were carried out using ECOTECT for creating the models and RADIANCE building analysis software packages for lighting analysis due to the compatibility between the two. ECOTECT is a building analysis software package used at the conceptual building design stage. RADIANCE is now the most widely accepted and used lighting simulation program for day-lighting research with several studies demonstrating good agreement with measured data confirming the validity of its use. Besides, it has great potential particularly in parametric studies where it allows for quick assessment of the chosen design variables.

The model simulated a square, five storeys, four sided top-lit atrium with full-scale dimensions of $16 \mathrm{~m}$ length $\times 16 \mathrm{~m}$ width $\times 20 \mathrm{~m}$ height corresponding to a WI of 1.25 . The adjoining spaces had a floor to floor height of $4 \mathrm{~m}$, with the false ceiling of $1 \mathrm{~m}$ at its underside for servicing, giving a clear height of $3 \mathrm{~m}$ and a room depth of $9 \mathrm{~m}$ as shown in Fig. 2.

All dimensions were defined in terms of interior envelope dimensions and did not include thicknesses of building elements, atrium roof, light scoops or light shelves in order to reduce the number of variables under consideration. Cut outs in the atrium facades were made to represent glazing positions, however no glazing was included. Reflectances of all surfaces were chosen specifically to represent real buildings; atrium walls and floor were assigned $85 \%$ and $40 \%$ reflectance, respectively, whilst the adjoining space walls, floor and ceiling were assigned $60 \%, 40 \%$ and $95 \%$ reflectances, respectively.

To establish the most effective distribution of windows, a pilot test was carried out to compare two window options as shown in Fig. 3 using Aschehoug's optimum ratio of 50\% glazing on the 4th floor, $60 \%$ glazing on the 3rd floor, $70 \%$ glazing on the 2nd floor and $100 \%$ glazing on the 1 st floor with all the above experimental set up, for a four storey building:

Window Option 1 - One continuous horizontal strip window with the top reveal at $3 \mathrm{~m}$ in line with the underside of the false ceiling centred in plan.
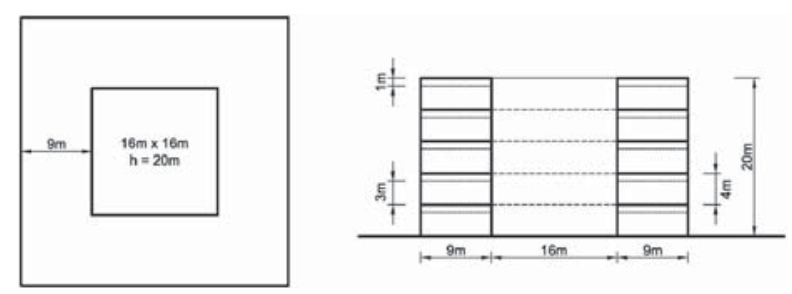

Figure 2: Plan and section of the atrium model.
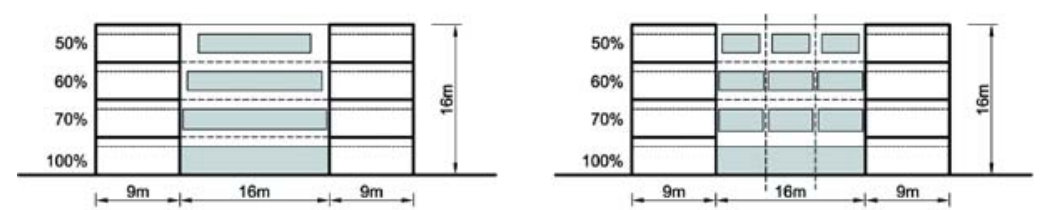

Figure 3: Pilot test of two window options - one continuous horizontal window and three vertical windows. 

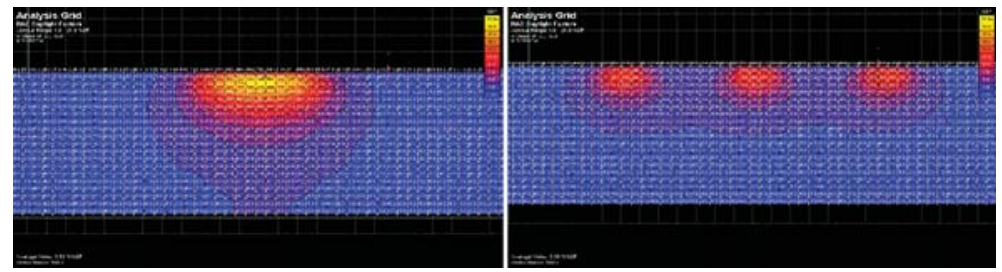

Figure 4: Daylight distribution in the adjoining spaces for one continuous horizontal window and three vertical windows.

Table 1: Three curve options of glazing ratios (each with five glazing ratios) for parametric modelling.

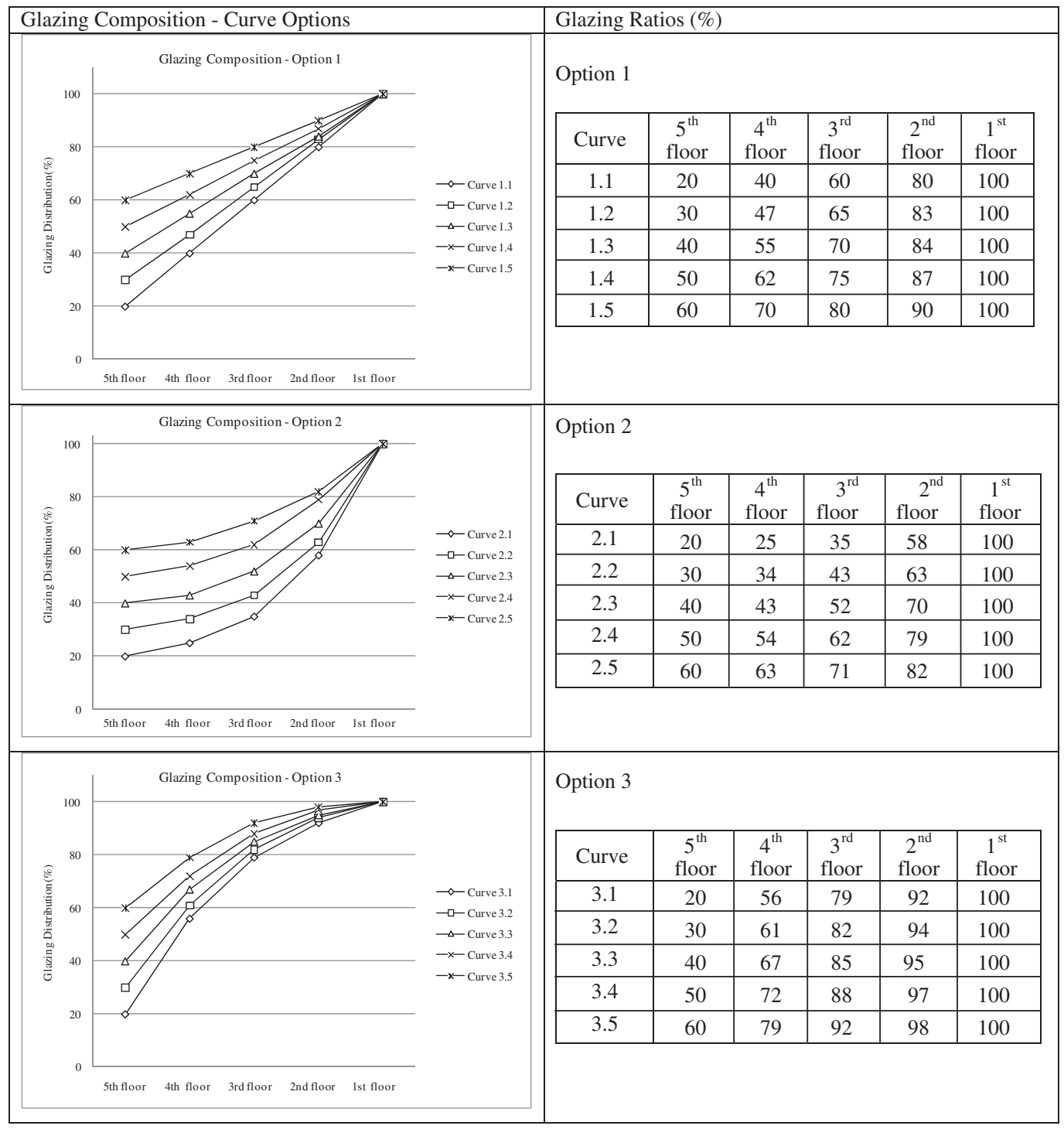


Window Option 2 - Three vertical windows with the top reveal at $3 \mathrm{~m}$ in line with the underside of the false ceiling centred in plan

The results of this comparison as shown in Fig. 4 demonstrated that the three vertical windows provided better distribution of light in the adjoining spaces and therefore this option was chosen for the parametric modelling of atrium facades.

Three curve options as shown in Table 1 were developed to establish the optimum glazing ratios and to assess if a particular nature of progressive increase in glazing affected daylight performance. As shown in the table, each curve option includes five options with $100 \%$ openings on the first floor, followed by progressive increase in openings for the intermediate floors, and $20 \%, 30 \%, 40 \%, 50 \%, 60 \%$ openings on top floor. The three curves were developed on the basis of the following:

Curve option 1 - A consistent and gradual increase in openings from top to bottom floors.

Curve option 2 - A shallow/slow increase in openings on the higher floors followed by a steep increase in openings on the lower floors.

Curve option 3 - A steep increase in openings on the higher floors followed by a shallow/slow increase in openings on the lower floors.

It was decided to analyse the worst case scenario, i.e. north facade of the south floor plate on all levels. Measurement points for DF calculations represented a working plane height of $0.85 \mathrm{~m}$ above the floor level. Horizontal DF measurements were taken for five positions on each floor: at the centre of the atrium, on the atrium wall, and 0.5, 3.2 and 5.8 m inside the adjoining space along its centre line and $6 \mathrm{~m}$ on either sides of it, in line with the centre of the three window openings. On completion of data input in ECOTECT, the models were exported to RADIANCE using the export manager tool for physical real lighting analysis.

\section{RESULTS AND FINDINGS}

Variation in façade compositions had no influence on DFs at $5.8 \mathrm{~m}$ into the adjoining space. On comparison of the three curve options, it is evident that there is a very good agreement between them. However, when results were compared with those obtained for curve option 1, curve option 3 gave marginally better DFs overall while curve option 2 gave slightly lower DFs for particular positions on third and fourth floor. This was mainly observed at $500 \mathrm{~mm}$ in the adjoining space when DFs for Curves 2.1, 2.2, 2.3 were 5-12\% lower and for Curves 3.1, 3.2, 3.3 were 1-15\% higher than those obtained by Curves 1.1, 1.2, 1.3, respectively.

For all the three curves, the options with $50 \%$ and $60 \%$ openings on the top floor (curves 1.4, 1.5, $2.4,2.5,3.4,3.5$ ) performed better than those with $20 \%, 30 \%, 40 \%$ openings on the top floor (curves $1.1,1.2,1.3,2.1,2.2,2.3,3.1,3.2,3.3)$.

Comparing data for the curves 1.4, 1.5, 2.4, 2.5, 3.4, 3.5 in the four positions (atrium centre, atrium wall, at 0.5 and $3.2 \mathrm{~m}$ in the adjoining space), DF values for the all three curves were very similar except at $500 \mathrm{~mm}$ into the adjoining space on the fourth and fifth floor when DFs for curves $1.4,2.4,3.4$ were lower by $3 \%$ to $6.6 \%$ than those for $1.5,2.5,3.5$ as shown in Fig. 5 . Curves 1.5 and 3.5 gave very similar results and were the best curves, however given that curve 3 also performed best for 3.1, 3.2, 3.3 conditions, it was concluded that curve 3.5 with $60 \%$ opening on top floor, $79 \%$ opening on fourth floor, $92 \%$ opening on third floor, $98 \%$ opening on second floor and $100 \%$ opening on ground floor was the best option.

Whilst 1.4, 1.5, 2.4, 2.5, 3.4, 3.5 curves performed much better than the other options, these curves gave very similar DFs suggesting that the different progressive increases have limited influence on daylight quantity and its distribution in the adjoining spaces. Therefore, given the simplicity of curve 1 , its performance, probability and ease of use in practice, further investigation of Curve 1 


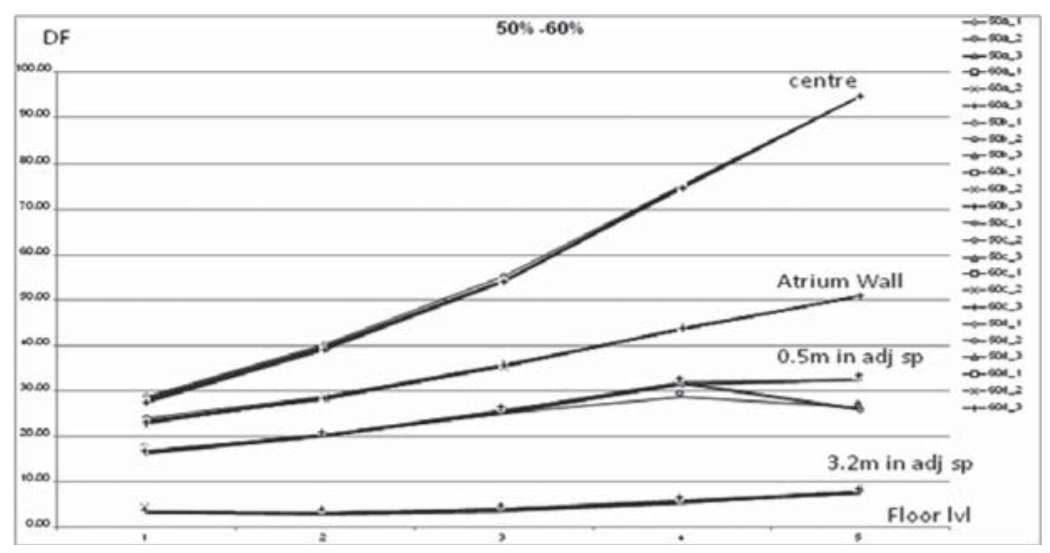

Figure 5: Comparison of DFs obtained for curves 1.4, 1.5, 2.4, 2.5, 3.4, 3.5.

was undertaken to understand its influence on daylight distribution in the atrium and its adjoining spaces on all floors.

Curves $1.1,1.2,1.3$ provide quite similar DFs within the range of approximately $2.6 \%$ difference for the various points with the exception of the top two floors where DFs improved with increase in window sizes particularly at $500 \mathrm{~mm}$ inside the adjoining space. Notably, in comparison with 1.1, DFs for 1.2 increased by $10 \%$ on the fourth floor at $500 \mathrm{~mm}$, and $2.3 \%$ on top floor at $3.2 \mathrm{~m}$ inside the adjoining space. This trend continued for option 1.3 when compared to 1.2 resulting in 5\% and $6.7 \% \mathrm{DF}$ increase on the fourth floor and top floor at $500 \mathrm{~mm}$ inside the adjoining space, respectively. Increase in openings on top floor from options 1.3 to 1.4 provided $3 \%$ increase on the fourth floor and $10.2 \%$ increase on top floor and for options 1.4 to 1.5 provided $6.6 \%$ increase on top floor at $500 \mathrm{~mm}$ inside the adjoining space. This demonstrates that the various options only affected DFs on the top two floors, mainly at $500 \mathrm{~mm}$ in the adjoining space, but did not improve DFs on the lower floors where more daylight is typically required.

When comparing option 1.1 with 1.5, DF at $500 \mathrm{~mm}$ inside the adjoining space for 1.5 improved significantly by $17.8 \%$ and $24.6 \%$ on fourth and fifth floor, respectively. For 1.5 , DF at $3 \mathrm{~m}$ inside the adjoining space also increased by $5.1 \%$ on the top floor and for all other points increase in DF ranged between $0.5 \%$ and $2.4 \%$. However, on the lower floors DFs were very similar for both the options and were in general higher by $0.1 \%$ and $2.6 \%$ for option 1.1 . This difference was mainly noted in the centre of the atrium position (1.8-2.6\%) suggesting that the two options have very little influence (0.1-0.9\% difference range in all other positions) on daylight availability in the lower adjoining floors. However, option 1.5 has significant influence on the top two floors and has the potential to affect daylight in these spaces.

When the difference in DFs for all the positions on five floors for the five façade compositions was compared as shown in Fig. 6, it was observed that the variation in façade composition has limited influence on DFs $(0.8-2.6 \%)$ at the centre of the atrium and on the atrium wall $(0.2-1.3 \%)$. Variation in façade composition has very little influence on DFs in the adjoining spaces except for the top two floors and was most pronounced at $0.5 \mathrm{~m}$ (17.8\% on 4th floor and $24.6 \%$ on the 5th floor) and $3.2 \mathrm{~m}$ $(5.1 \%)$ on the top floor adjoining space. Façade compositions have almost no influence on the lower floor adjoining spaces at $3 \mathrm{~m}$ and beyond.

For all the five curves, DF at the atrium centre dropped by $65.9-66.9 \%$ from top to bottom floor, and DF at the atrium wall dropped by $25.7-28.1 \%$ from top to bottom floor. Whilst progressive 


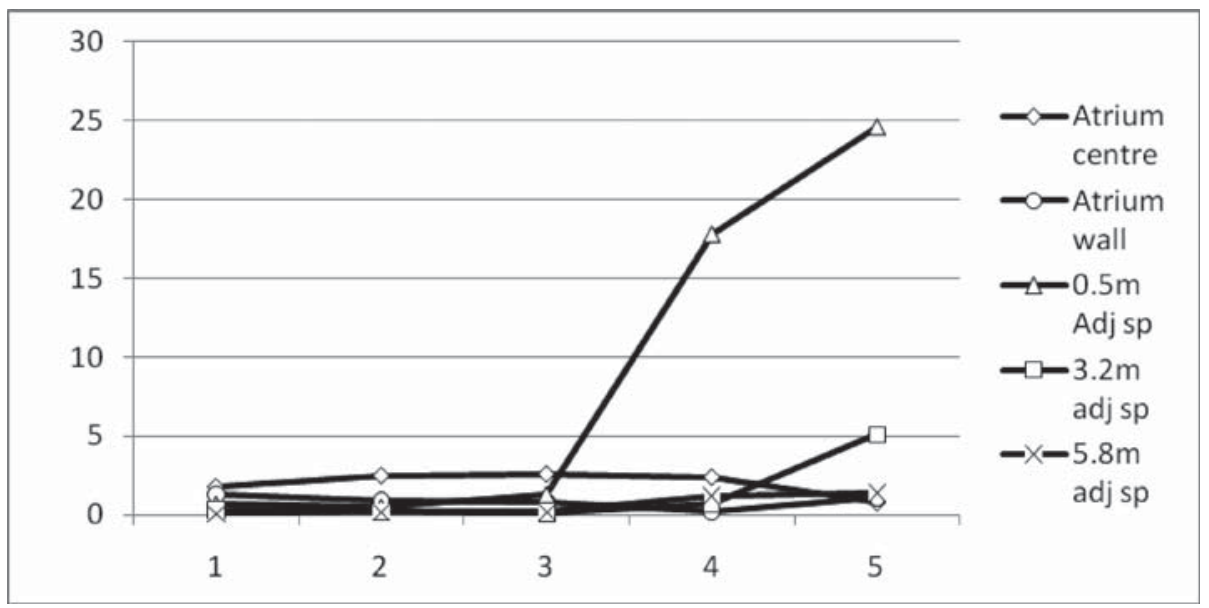

Figure 6: Difference in DFs for all positions for the five façade compositions $(1.1,1.2,1.3,1.4$, and 1.5).

increase in windows caused DFs at $500 \mathrm{~mm}$ in the adjoining space to increase by $9.3 \%, 8.4 \%$ and $1.2 \%$ from top to bottom floor for 1.1, 1.2 and 1.3 options, respectively, the same position DFs fell by $6.6 \%$ and $15.6 \%$ from top to bottom floor for 1.4 and 1.5 options respectively. For option 1.1, DFs from top to bottom floor increased by $1.0 \%$ at $3.2 \mathrm{~m}$, however for all the other options DFs from top to bottom floor decreased by $1.2-4.3 \%$ at $3.2 \mathrm{~m}$ and was more prominent for curves 1.5 and 1.4 where the DFs dropped by over 4\%. Whilst the drop in DFs on the lower floors for 1.4 and 1.5 might appear to be a negative effect, in reality it is only an increased difference between DF values between the top and bottom floors due to the increase in DFs on the top floors as a result of larger openings whilst maintaining DFs on the lower floors to those obtained by the other options. DFs decreased from top to bottom floor by $0.6-1.1 \%$ at $5.8 \mathrm{~m}$ in the adjoining space for $1.4,1.3$, $1.2,1.1$ options.

Figure 7 shows the minimum and maximum difference in DF observed and the rate of decay on each floor from atrium centre to $3.2 \mathrm{~m}$ in the adjoining space for the five options. It is evident that the horizontal DFs significantly vary in different positions higher up in the atrium but this difference gradually reduces from top to bottom floors. Additionally, there is a better agreement in DFs obtained from the five options $(1.1,1.2,1.3,1.4,1.5)$ lower down in the atrium than on the upper floors demonstrating that the effect of façade compositions is limited on the lower floors and distribution of light for all the five options on the lower three floors is similar.

For all the five options, on level 1 the drop in DF ranges between $4 \%$ and $5 \%$ from centre to atrium wall position, $11-12 \%$ from centre to $0.5 \mathrm{~m}$ in the adjoining space and $\sim 13 \%$ from 0.5 to 3.2 $\mathrm{m}$ in the adjoining space. On level 2, DF drops by 11-13\% from centre to atrium wall position, $19-21 \%$ from centre to $0.5 \mathrm{~m}$ in the adjoining space and $\sim 17 \%$ from 0.5 to $3.2 \mathrm{~m}$ in the adjoining space. On level 3, DF drops by 18-20\% from centre to atrium wall position, 28-32\% from centre to $0.5 \mathrm{~m}$ in the adjoining space and $20-22 \%$ from 0.5 to $3.2 \mathrm{~m}$ in the adjoining space. On level 4 and 5 , DF drops by $31-33 \%$ and $44-46 \%$ from centre to atrium wall position; $43-63 \%$ and $62-88 \%$ from centre to $0.5 \mathrm{~m}$ in the adjoining space, and $\sim 9-26 \%$ and $5-25 \%$ from 0.5 to $3.2 \mathrm{~m}$ in the adjoining space, respectively. There is a very big drop in DFs from centre to $0.5 \mathrm{~m}$ in the adjoining space on levels 4 and 5 for option 1.1 due to very small windows on this floor but the DF drop reduces further into the adjoining space due to reduction in available light at $3.2 \mathrm{~m}$ in the space. Whilst there is a 


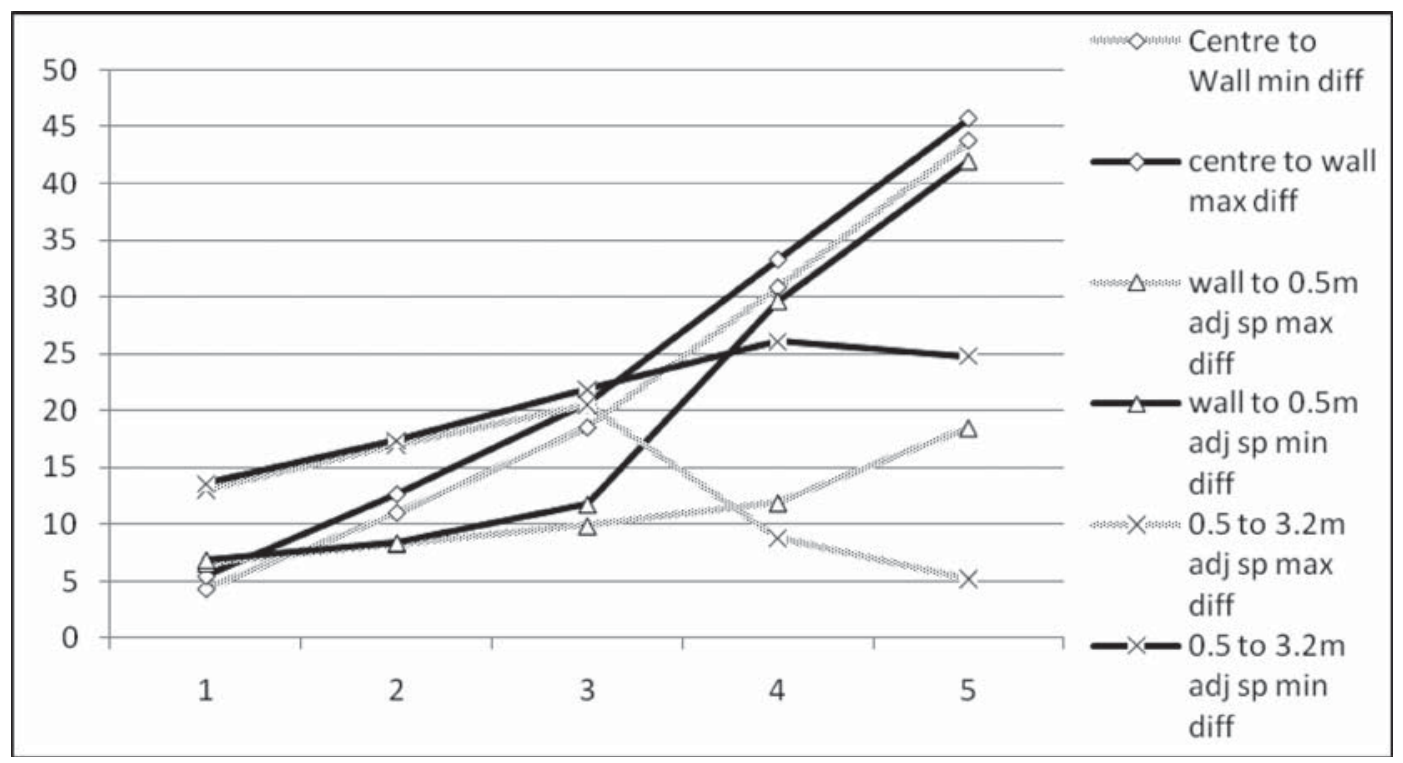

Figure 7: Distribution in DF on each floor from atrium centre to $3.2 \mathrm{~m}$ in the adjoining space for the five options.

bigger drop for option 1.5 from $0.5 \mathrm{~m}$ to $3.1 \mathrm{~m}$ into the adjoining space on the top two floors, the overall light levels are much higher.

\section{CONCLUSIONS}

This paper examined the influence of an atrium building's interior envelope design on indoor environmental quality (IEQ) to provide a sustainable daylight strategy and to improve the eco-dynamics of this building type.

The study concluded that for a medium sized building progressive increase in glazing from top to bottom floor has limited influence on daylight in the atrium space and its adjoining spaces allowing flexibility in façade design, whilst maintaining the design integrity and general trend in the ratios.

Variation in façade compositions has limited influence on DFs $(0.8-2.6 \%)$ at the centre of the atrium and on the atrium wall $(0.2-1.1 \%)$. Façade compositions have almost no influence on the lower floor adjoining spaces where more daylight is typically required, but can have significant influence on the top two floors.

Having smaller windows on the top floor (20\%) increases DFs in the adjoining spaces; however, this increase is not significant and compromises DFs on the top two floors. However, a more gradual increase in windows with $50 \%, 60 \%$ openings on top floor, increasing up to $100 \%$ on the lowest floor increases DFs on the top floors without significantly compromising DFs on the lower floors.

When comparing the $20 \%$ with the $60 \%$ option of windows on top floor, DF at $0.5 \mathrm{~m}$ inside the adjoining space improved significantly by $17.8 \%$ and $24.6 \%$ on 4 th and 5 th floor, respectively. DF at $3 \mathrm{~m}$ inside the adjoining space also increased by $5 \%$ on the top floor and for all other points increase in DF ranged between $0.5 \%$ and $2.4 \%$.

In this study, for a five storey building with a WI of 0.8 , curve option 3 with $60 \%$ opening on top floor, $79 \%$ opening on fourth floor, $92 \%$ opening on third floor, $98 \%$ opening on second floor and $100 \%$ opening on ground floor performed the best. 
Whilst the drop in DFs on the lower floors for the $50 \%$ and $60 \%$ options might appear to be a negative effect, in reality it is only an increased difference between DF values between the top and bottom floors due to the increase in DFs on the top floors as a result of larger openings whilst maintaining DFs on the lower floors to those obtained by the other options $(20 \%, 30 \%, 40 \%)$.

At $5.8 \mathrm{~m}$ inside the adjoining space, DFs were nearly the same for all the options but varied slightly for $3.2 \mathrm{~m}$ and more significantly at $0.5 \mathrm{~m}$ into the space, suggesting that glazing distributions do not affect DFs beyond $3 \mathrm{~m}$ into the adjoining space. Horizontal DFs significantly reduce from centre to the atrium wall and drop further into the adjoining space. This effect is more noticeable on the upper floors; however, the rate of decay gradually reduces from top to bottom floors.

This study could be extended to different atrium types - three sided, linear, and stepped atria to assess the performance of the chosen glazing ratio options in different geometries. Further experiments could also be conducted to include different types of wall fenestrations, glazing types including low emissivity glass, shading devices, light shelves and roof systems to assess their impact on available light in an atrium and its adjoining spaces. Additionally, it is vital to link theory and practice through comparing building simulation studies with existing case studies. Finally, more integrated studies of contrasting performance variables such as daylight, thermal, and acoustic, alongside energy use data are required that would provide guidelines for improved energy efficiencies and enable high performance buildings.

\section{ACKNOWLEDGMENTS}

The author would like to thank Mrs Sheetal Merai for her contribution in parametric modelling of this research project. Sheetal completed her Masters in Architecture, at the University of Nottingham in 2008 and currently works in a private architectural practice in Leeds.

\section{REFERENCES}

[1] The Chartered Institution of Building Services Engineers, CIBSE Code For Interior Lighting, CIBSE: London, 1984.

[2] Letherman, K.M. \& Wright, J.C., Illuminance in Atria: review of prediction methods. Lighting Research And Technology, 30(1), pp. 1-11, 1998. doi:10.1177/096032719803000101

[3] Aizlewood, M.E., Butt, J.D., Isaac, K.A. \& Littlefair, P.J., Daylight in Atria: A Comparison of Measurements, Theory and Simulation LUX EUROPA, Amsterdam, pp. 571-584, 1997.

[4] Sharples, S. \& Lash, D., Daylight in atrium buildings: a critical review Architectural Science Review, 50(4), pp. 301-312, 2007.

[5] Oretskin, B.L., Studying the efficiency of lightwells by means of models under an artificial sky. Proceedings of the 7th Ases Passive Conference, Knoxville, TX, pp. 459-463, 1982.

[6] Navvab, M. \& Selkowitz, S., Daylighting data for atrium design. Proceedings, 9th National Passive Solar Conference, Columbus, pp. 495-500, 1984.

[7] Cartwright, V., The use of lightwells as a daylight strategy. Proceedings '85, American SES, Raleigh, NC, pp. 115-118, 1985.

[8] Aschehoug, O., Daylight design for glazed spaces. Proceedings From The International Daylighting Conference 2, Long Beach, CA, November, pp. 237-243, 1986.

[9] Liu, A., Navvab, M. \& Jones, J., Geometric shape index for daylight distribution variations in atrium spaces. Proceedings of the 16th National Passive Solar Conference, American Solar Energy Society, Denver, 1991.

[10] Cole, R.J., The effect of the surfaces enclosing atria on the daylight in adjacent spaces. Building and Environment, 25(1), pp. 37-42, 1990. doi:10.1016/0360-1323(90)90039-T 
[11] Boubekri, M., The effect of the cover and reflective properties of a four sided atrium on the behaviour of light. Architectural Science Review, 38(3), pp. 3-8, 1995.

[12] Willbold-Lohr, G., Daylighting in glazed atria. Proceedings of the 2nd European Conference on Architecture, Paris, pp. 16-20, 1989.

[13] Iyer, R.U., Daylighting in atrium spaces. Architectural Science Review, (37), pp. 195-208, 1994.

[14] Matusiak, B., Aschehoug, O. \& Littlefair, P., Daylighting strategies for an infinitely long atrium: an experimental evaluation. Lighting Research And Technology, 31(1), pp. 23-34, 1999. doi:10.1177/096032719903100105

[15] Sharples, S. \& Mahambrey, S., Reflectance distributions and atrium daylight levels: a model study Lighting Research \& Technology, 31(4), pp. 165-170, 1999. doi:10.1177/096032719903100405

[16] Samant, S. \& Sharples, S. \& Surface reflections distributions and their effect on average daylight factor values in atrium buildings. Architectural Science Review, 47(2), pp. 177-182, 2004.

[17] Littlefair, P., Daylight prediction in atrium buildings, Solar Energy, 73(2), pp. 105-109, 2002. doi:10.1016/S0038-092X(02)00038-5

[18] Sharples, S. \& Lash, D., Reflectance distributions and vertical daylight levels in atria Lighting Research \& Technology, 36(1), pp. 45-57, 2004. doi:10.1191/1477153504li103oa

[19] Matusiak, B., The impact of lighting/daylighting and reflectances on the size impression of the room. Full-scale studies Architectural Science Review, pp. 47(2), 2004.

[20] Calcagni, B. \& Paroncini, M., Daylight factor prediction in atria building designs. Solar Energy, 76 (6), pp. 669-682, 2004. doi:10.1016/j.solener.2004.01.009

[21] Samant, S. \& Yang, F., Daylighting in atrium: the effect of atrium geometry and reflectance distributions. International Journal of Lighting Research and Technology, 39(2), pp. 147-158, 2007. doi:10.1177/1365782806074482

[22] Lau, B. \& Duan, Z., The daylight benefit conferred upon adjoining rooms by specular surfaces in top-lit atria Architectural Science Review, 51(3), pp. 204-211, 2008. 